\title{
Relação entre o tempo, o tipo e o efeito do ataque no Voleibol masculino juvenil de alto nível competitivo
}

\author{
Relationship between tempo, type and effect of attack in male \\ young volleyball players from in high competitive level
}

\author{
Gustavo Costa 1 \\ Isabel Mesquita 2 \\ Pablo Juan Greco 2 \\ Natália Neiva Ferreira ${ }^{3}$ \\ José Cícero Moraes ${ }^{4}$
}

1. Minas Tênis Clube. Núcleo de Integração de Ciências do Esporte. Belo Horizonte. MG, Brasil.

2. Universidade do Porto, Faculdade de Desporto. Porto, Portugal.

3. Universidade Federal de Minas Gerais. Departamento de Educação Física. Belo Horizonte. MG, Brasil.

4. Universidade Federal do Rio Grande do Sul. Porto Alegre, RS. Brasil

Recebido em 08/02/10 Revisado em 23/03/10 Aprovado em 27/05/10
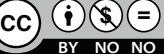

Licença: Creative Commom
Resumo - Atualmente, no Voleibol de alto nível, é inquestionável o papel que o ataque assume no desenrolar do jogo e no rendimento das equipes, o que requer ataques rápidos, precisos e potentes, exigindo, para tal, o domínio técnico e a capacidade de adaptação aos constrangimentos situacionais do jogo. Desta forma, o objetivo deste trabalho foi o de analisar a relação entre o tempo e o tipo de ataque com o efeito do ataque em seleções nacionais juvenis de Voleibol masculino. Para tal, recorreu-se à análise de 1191 ações de ataque de seleções nacionais presentes no Campeonato Mundial Juvenil. Verificou-se que o efeito de ataque mais recorrente foi o ponto, sendo que os ataques mais rápidos apresentaram maior frequência de acontecimento, assim como os ataques potentes. $\mathrm{O}$ jogo mais rápido favoreceu a obtenção do ponto e o ataque potente emanou da necessidade de sobrepor-se ao sistema defensivo adversário.

Palavras-chave: Voleibol; Tempo de ataque; Tipo de ataque; Efeito do Ataque.

Abstract - At present, the role the attack plays in a high level volleyball game and team performance is unquestionable. This sports modality is characterized by fast, precise and powerful attacks that require technical control and the ability to adapt to situational constraints of the game. Thus, the objective of this study was to examine the relationship between time and type of attack and the effect of the attack in national junior male volleyball teams. For this purpose, 1191 attack actions of national teams participating in the World Junior Championship were analyzed. The most recurrent effect of attack was the point, and faster and powerful attacks occurred more frequently. The fastest game favored gaining of a point and the powerful attack emerged from the need to overlap the opponent's defensive system.

Key words: Volleyball; Time of attack; Type of attack; Effect of attack. 


\section{INTRODUÇÃO}

A análise de jogo é imprescindível no desenvolvimento das modalidades esportivas coletivas ${ }^{1-3}$, permitindo a escolha de indicadores pertinentes acerca da prestação dos jogadores em contextos específicos ${ }^{4-6}$.

Atualmente, no Voleibol de alto nível de rendimento competitivo, é inquestionável o papel que o ataque assume no desenrolar do jogo e no rendimento das equipes, demonstrando elevada correlação com a vitória no jogo ${ }^{7-12}$. Face ao elevado nível de desempenho das equipes, a sua importância tornou-se mais relevante ${ }^{13}$, sendo preciso atacar mais alto, com maior potência e precisão, exigindo para tal, o domínio técnico e a capacidade de adaptação aos constrangimentos situacionais do jogo ${ }^{14}$.

As tendências evolutivas do Voleibol demonstram que o jogo mais rápido é determinante no sucesso do ataque ${ }^{15-21}$, ao mesmo tempo em que se torna essencial diminuir a utilização do ataque mais lento $\left(3^{\circ} \text { tempo }\right)^{22}$. Palao, Santos e Ureña ${ }^{11}$, num estudo realizado com trinta e três jogos das equipes masculinas presentes nos Jogos Olímpicos de Sydney, verificaram que as equipes melhores classificadas se distinguiram, principalmente, pelo desempenho mais elevado ao nível do ataque. Rocha e Barbanti ${ }^{18}$, analisaram vinte jogos de Voleibol masculino de alto nível, referentes à Superliga ${ }^{\circledR}$ de 1999/2000, e perceberam que o $1^{\circ}$ tempo de ataque (o mais rápido) apresentou eficácia superior aos $2^{\circ}$ e $3^{\circ}$ tempo (intermédio e menos rápido respectivamente); foram realizados mais ataques de $1^{\circ}$ e $2^{\circ}$ tempo e conforme a velocidade do levantamento foi diminuindo, foram-se reduzindo as chances de ponto pelo ataque.

No Voleibol, as condições de organização das ações distinguem-se em função dos constrangimentos situacionais, sendo que o efeito do ataque mostra estar dependente do tipo de ataque, nomeadamente da sua trajetória, da interferência do bloqueio e da sua potência ${ }^{23}$.

O tipo de ataque mais comum é o forte, estando a sua utilização dependente das especificidades emergentes relacionadas com o sistema defensivo adversário $^{18}$. Castro e Mesquita ${ }^{18}$ analisaram doze jogos da Liga Mundial Masculina e seis jogos da fase final do Campeonato da Europa Masculino de 2005, e observaram que houve o predomínio do ataque forte, devido às boas condições de finalização do ataque proporcionadas pela debilidade do sistema defensivo adversário.

A pesquisa sobre estas variáveis tem utilizado, preferencialmente, equipes adultas de voleibol de alto rendimento. Dada a escassez de estudos aplicados em equipes de base, caracterizadores do rendimento em competição, revela-se pertinente realizar pesquisa nesse âmbito, contribuindo, assim, com informação pertinente para o treinamento. O presente estudo pretende analisar a natureza da relação entre o tempo e o tipo de ataque sobre o seu efeito no âmbito da categoria juvenil masculina de alto rendimento competitivo.

\section{PROCEDIMENTOS METODOLÓGICOS}

\section{Amostra}

O presente estudo teve como amostra um conjunto de seleções nacionais presentes no Campeonato Mundial Juvenil Masculino de 2007 (Brasil, Rússia, Iran, Itália, Argentina, Bulgária, Estados Unidos, Cuba, Eslovênia, Egito e Japão). Recorreu-se à observação de 11 jogos, obtendo-se um total de 1191 ações de ataque.

\section{Variáveis do estudo}

Efeito do ataque: Analisou-se o efeito do ataque sobre o sistema defensivo adversário (tabela 1). $\mathrm{O}$ instrumento de observação aplicado foi a adaptação da escala de Eom e Schutz ${ }^{7-8}$.

Tabela 1. Categorias e escalas de avaliação do efeito do ataque

\begin{tabular}{ll}
\hline Escala & \\
\hline 0 & $\begin{array}{l}\text { Erro do atacante } \\
\text { Erro do atacante decorrente do bloqueio } \\
1\end{array}$ \\
2 & $\begin{array}{l}\text { Continuidade - defesa com contra-ataque } \\
\text { organizado } \\
\text { Continuidade - Rebatida pelo bloqueio } \\
\text { para a equipe adversária }\end{array}$ \\
3 & $\begin{array}{l}\text { Continuidade - Defesa sem contra-ataque } \\
\text { organizado }\end{array}$ \\
5 & Ponto de ataque \\
\hline
\end{tabular}

Tempo de Ataque - Para esta categoria, adotou-se a classificação de Sellinger, Ackermann-Blount ${ }^{24}$, a qual estabelece uma relação entre o momento de contato da bola pelo passador e a saída para o ataque do atacante. Deste modo, obtiveram-se as seguintes categorias: $1^{\circ}$ tempo de ataque: o atacante contata a bola depois do levantador soltá-la; $2^{\circ}$ tempo de ataque: o atacante sai para o ataque quando a bola chega às mãos do levantador; $3^{\circ}$ tempo de ataque: o atacante sai para o ataque quando a bola chega ao ponto mais alto da trajetória ascendente depois de sair das mãos do levantador.

Tipo de ataque realizado: Através da literatura consultada, verificaram-se várias classificações dos tipos de ataque, de acordo com critérios distintos (direção do ataque, tipo de corrida de aproximação, 
exploração do bloco, etc). No presente estudo, utilizou-se à adaptação de Weishoff ${ }^{23}$ para analisar o tipo de ataque realizado, dada a variabilidade de opções técnicas que comporta:

1) ataque potente na paralela: ataque forte dirigido paralelamente à linha lateral da quadra;

2) ataque potente na diagonal aberta: ataque forte para a grande diagonal situado no fundo da quadra;

3) ataque potente na diagonal fechada: ataque forte para a pequena diagonal, entre os dois defensores da linha defensiva e da linha ofensiva ou linha dos três metros;

4) ataque potente frontal: ataque forte no sentido da corrida de aproximação;

5) amorti: corresponde aos ataques em que a bola é contactada na parte inferior com a ponta dos dedos ou superior através de um movimento rápido de pulso, de forma a impulsionar a bola para baixo na direção do campo adversário.

6) ataque colocado: a bola é contactada na parte inferior, com a mão em "concha" e os dedos bem afastados. Os jogadores executam um movimento enrolado com o pulso para imprimir rotatividade à bola;

7) block out: o atacante procura explorar as fraquezas do bloqueio adversário, atacando a bola de encontro a este, para que esta ressalte para fora da quadra ou de encontro à vareta;

8) costura: o atacante procura explorar o espaço entre os bloqueadores;
9) outros: é o ataque realizado através da manchete, toque de dedos, etc.

Procedimento de recolha de dados Os jogos foram filmados de "topo", ou seja, a visualização do campo longitudinalmente, possibilitando observar e recolher informação acerca das variáveis em estudo.

Procedimentos estatísticos e de fiabilidade

Para a análise exploratória, recorreu-se à estatística descritiva, tendo-se obtido as frequências e respectivas percentagens para cada uma das categorias das variáveis em estudo (tempo de ataque e tipo de ataque). Para a associação entre as variáveis estudadas, recorreu-se ao teste do Qui-Quadrado, com a correção de Monte Carlo, sempre que menos de 20\% das células apresentaram valor inferior a 5.

Para o estudo da fiabilidade, foram analisadas $34,9 \%$ das ações, valores substancialmente superiores aos de referência (10\%), apontados pela literatura ${ }^{25}$. A fiabilidade intraobservador e interobservador mostrou valores de Kappa entre 0.82 e 1 para todas as variáveis, substancialmente superiores aos valores mínimos aceitáveis apontados pela literatura $(0.75)^{26}$.

\section{RESULTADOS}

O tempo de ataque mostrou estar associado ao efeito do ataque $\left(\mathrm{X}^{2}=60,472 ; \mathrm{p}=0,000\right)$ (tabela 2).

Considerando o tempo de ataque (tabela 2 ),

Tabela 2. Relação entre o tempo e o efeito do ataque.




Tabela 3. Relação entre o tipo e o efeito do ataque.

\begin{tabular}{|c|c|c|c|c|c|c|c|c|}
\hline \multirow{2}{*}{ Tipo de ataque } & & \multicolumn{7}{|c|}{ Efeito do ataque } \\
\hline & & 0 & 1 & 2 & 3 & 4 & 5 & Total \\
\hline \multirow{5}{*}{$\begin{array}{l}\text { Ataque potente na } \\
\text { paralela }\end{array}$} & Ocorrido & 34 & 26 & 4 & 2 & 30 & 71 & 167 \\
\hline & Esperado & 17,2 & 15,7 & 20,1 & 6,5 & 31,8 & 75,7 & 167,0 \\
\hline & \%Tip ataq & $20,4 \%$ & $15,6 \%$ & $2,4 \%$ & $1,2 \%$ & $18,0 \%$ & $42,5 \%$ & $100,0 \%$ \\
\hline & $\%$ Efi ataq & $27,6 \%$ & $23,2 \%$ & $2,8 \%$ & $4,3 \%$ & $13,2 \%$ & $13,1 \%$ & $14,0 \%$ \\
\hline & Ajust Res. & 4,6 & 2,9 & $-4,1$ & $-1,9$ &,- 4 &,- 8 & \\
\hline \multirow{5}{*}{$\begin{array}{l}\text { Ataque potente na } \\
\text { diagonal aberta }\end{array}$} & Ocorrido & 24 & 18 & 14 & 5 & 52 & 165 & 278 \\
\hline & Esperado & 28,7 & 26,1 & 33,4 & 10,7 & 53,0 & 126,0 & 278,0 \\
\hline & \%Tip ataq & $8,6 \%$ & $6,5 \%$ & $5,0 \%$ & $1,8 \%$ & $18,7 \%$ & $59,4 \%$ & $100,0 \%$ \\
\hline & $\%$ Efi ataq & $19,5 \%$ & $16,1 \%$ & $9,8 \%$ & $10,9 \%$ & $22,9 \%$ & $30,6 \%$ & $23,3 \%$ \\
\hline & Ajust Res. & $-1,1$ & $-1,9$ & $-4,1$ & $-2,0$ &,- 2 & 5,4 & \\
\hline \multirow{5}{*}{$\begin{array}{l}\text { Ataque potente na } \\
\text { diagonal fechada }\end{array}$} & Ocorrido & 7 & 0 & 2 & 0 & 6 & 32 & 47 \\
\hline & Esperado & 4,9 & 4,4 & 5,6 & 1,8 & 9,0 & 21,3 & 47,0 \\
\hline & \%Tip ataq & $14,9 \%$ &, $0 \%$ & $4,3 \%$ &, $0 \%$ & $12,8 \%$ & $68,1 \%$ & $100,0 \%$ \\
\hline & $\%$ Efi ataq & $5,7 \%$ &, $0 \%$ & $1,4 \%$ &, $0 \%$ & $2,6 \%$ & $5,9 \%$ & $3,9 \%$ \\
\hline & Ajust Res. & 1,0 & $-2,3$ & $-1,7$ & $-1,4$ & $-1,1$ & 3,2 & \\
\hline \multirow{5}{*}{$\begin{array}{l}\text { Ataque potente } \\
\text { frontal }\end{array}$} & Ocorrido & 35 & 55 & 17 & 36 & 69 & 81 & 293 \\
\hline & Esperado & 30,3 & 27,6 & 35,2 & 11,3 & 55,8 & 132,8 & 293,0 \\
\hline & \%Tip ataq & $11,9 \%$ & $18,8 \%$ & $5,8 \%$ & $12,3 \%$ & $23,5 \%$ & $27,6 \%$ & $100,0 \%$ \\
\hline & $\%$ Efi ataq & $28,5 \%$ & $49,1 \%$ & $11,9 \%$ & $78,3 \%$ & $30,4 \%$ & $15,0 \%$ & $24,6 \%$ \\
\hline & Ajust Res. & 1,0 & 6,3 & $-3,8$ & 8,6 & 2,3 & $-7,0$ & \\
\hline \multirow{5}{*}{ Amorti } & Ocorrido & 10 & 3 & 17 & 1 & 19 & 17 & 67 \\
\hline & Esperado & 6,9 & 6,3 & 8,0 & 2,6 & 12,8 & 30,4 & 67,0 \\
\hline & \%Tip ataq & $14,9 \%$ & $4,5 \%$ & $25,4 \%$ & $1,5 \%$ & $28,4 \%$ & $25,4 \%$ & $100,0 \%$ \\
\hline & $\%$ Efi ataq & $8,1 \%$ & $2,7 \%$ & $11,9 \%$ & $2,2 \%$ & $8,4 \%$ & $3,1 \%$ & $5,6 \%$ \\
\hline & Ajust Res. & 1,3 & $-1,4$ & 3,5 & $-1,0$ & 2,0 & $-3,4$ & \\
\hline \multirow{5}{*}{ Ataque colocado } & Ocorrido & 10 & 7 & 42 & 1 & 44 & 25 & 129 \\
\hline & Esperado & 13,3 & 12,1 & 15,5 & 5,0 & 24,6 & 58,5 & 129,0 \\
\hline & \%Tip ataq & $7,8 \%$ & $5,4 \%$ & $32,6 \%$ &, $8 \%$ & $34,1 \%$ & $19,4 \%$ & $100,0 \%$ \\
\hline & $\%$ Efi ataq & $8,1 \%$ & $6,3 \%$ & $29,4 \%$ & $2,2 \%$ & $19,4 \%$ & $4,6 \%$ & $10,8 \%$ \\
\hline & Ajust Res. & $-1,0$ & $-1,6$ & 7,6 & $-1,9$ & 4,6 & $-6,3$ & \\
\hline \multirow{5}{*}{$\begin{array}{l}\text { Ataque explorando o } \\
\text { bloqueio (block out) }\end{array}$} & Ocorrido & 0 & 0 & 0 & 0 & 0 & 127 & 127 \\
\hline & Esperado & 13,1 & 11,9 & 15,2 & 4,9 & 24,2 & 57,6 & 127,0 \\
\hline & $\%$ Tip ataq &, $0 \%$ &, $0 \%$ &, $0 \%$ &, $0 \%$ &, $0 \%$ & $100 \%$ & $100,0 \%$ \\
\hline & $\%$ Efi ataq &, $0 \%$ &, $0 \%$ &, $0 \%$ &, $0 \%$ &, $0 \%$ & $23,5 \%$ & $10,7 \%$ \\
\hline & Ajust Res. & $-4,0$ & $-3,8$ & $-4,4$ & $-2,4$ & $-5,8$ & 13,1 & \\
\hline \multirow{5}{*}{$\begin{array}{l}\text { Ataque explorando o } \\
\text { bloqueio (costura) }\end{array}$} & Ocorrido & 0 & 0 & 1 & 0 & 0 & 15 & 16 \\
\hline & Esperado & 1,7 & 1,5 & 1,9 & 6 & 3,0 & 7,3 & 16,0 \\
\hline & \%Tip ataq &, $0 \%$ &, $0 \%$ & $6,3 \%$ &, $0 \%$ &, $0 \%$ & $93,8 \%$ & $100,0 \%$ \\
\hline & $\%$ Efi ataq &, $0 \%$ &, $0 \%$ &, $7 \%$ &, $0 \%$ &, $0 \%$ & $2,8 \%$ & $1,3 \%$ \\
\hline & Ajust Res. & $-1,4$ & $-1,3$ &,- 7 &,- 8 & $-2,0$ & 3,9 & \\
\hline \multirow{5}{*}{ Ataque outros } & Ocorrido & 3 & 3 & 46 & 1 & 7 & 7 & 67 \\
\hline & Esperado & 6,9 & 6,3 & 8,0 & 2,6 & 12,8 & 30,4 & 67,0 \\
\hline & \%Tip ataq & $4,5 \%$ & $4,5 \%$ & $68,7 \%$ & $1,5 \%$ & $10,4 \%$ & $10,4 \%$ & $100,0 \%$ \\
\hline & $\%$ Efi ataq & $2,4 \%$ & $2,7 \%$ & $32,2 \%$ & $2,2 \%$ & $3,1 \%$ & $1,3 \%$ & $5,6 \%$ \\
\hline & Ajust Res. & $-1,6$ & $-1,4$ & 14,7 & $-1,0$ & $-1,8$ & $-5,9$ & \\
\hline \multirow{4}{*}{ Total } & Ocorrido & 123 & 112 & 143 & 46 & 227 & 540 & 1191 \\
\hline & Esperado & 123,0 & 112,0 & 143,0 & 46,0 & 227,0 & 540,0 & 1191,0 \\
\hline & \%Tip ataq & $10,3 \%$ & $9,4 \%$ & $12,0 \%$ & $3,9 \%$ & $19,1 \%$ & $45,3 \%$ & $100,0 \%$ \\
\hline & $\%$ Efi ataq & $100 \%$ & $100 \%$ & $100 \%$ & $100 \%$ & $100 \%$ & $100 \%$ & $100,0 \%$ \\
\hline
\end{tabular}


observou-se que o ponto de ataque ocorreu mais do que o esperado no $1^{\circ}$ e $2^{\circ}$ tempo e menos no $3^{\circ}$ tempo de ataque. $\mathrm{O}$ ataque que permitiu defesa e contra ataque organizado, ocorreu mais do que o esperado após o $3^{\circ}$ tempo de ataque e menos no $1^{\circ}$ e $2^{\circ}$ tempo de ataque. Além disso, o ataque de $1^{\circ}$ tempo permitiu que ocorresse menos do que era esperado o bloqueio do adversário.

$\backslash$ Em termos de tempo de ataque, o mais frequente foi o $2^{\circ}$ tempo, seguido do $3^{\circ}$ tempo e por fim do $1^{\circ}$ tempo. Em referência ao efeito, o mais frequente foi o ponto do ataque.

$\mathrm{Na}$ análise do tipo de ataque (tabela 3), verificou-se que o ataque potente foi mais recorrente, com $65,8 \%$ de ocorrência, seguido pelo ataque colocado (16,4\%), ataque explorando o bloqueio (12\%) e ataque outros (5,6\%).

Do mesmo modo, o tipo de ataque também mostrou estar associado ao efeito do ataque $\left(\mathrm{X}^{2}=681,832 ; \mathrm{p}=0,000\right)$, tendo muitas das células contribuído para esta associação.

$\mathrm{O}$ ataque que culminou em ponto ocorreu mais do que o esperado através do ataque potente na diagonal aberta, do ataque potente na diagonal fechada, do block out e do costura e menos após o ataque potente frontal, o amorti, o ataque colocado e outros.

$O$ ataque que não permitiu a defesa com contra-ataque organizado ocorreu mais do que o esperado através do ataque potente frontal, o amorti e o ataque colocado e menos quando foi realizado pelo block out e pelo costura. $O$ ataque que foi rebatido pelo bloqueio para a defesa adversária, ocorreu mais do que o esperado pelo recurso do ataque potente frontal e menos após a realização do ataque potente na diagonal aberta e do block out. $\mathrm{O}$ efeito do ataque que permitiu defesa e contra-ataque organizado, ocorreu mais do que o esperado após o ataque colocado e o ataque outros e menos após o ataque potente na paralela, o ataque potente na diagonal aberta, o ataque potente frontal e o block out. Relativamente ao bloqueio do ataque, este ocorreu mais do que o esperado após o ataque potente na paralela e o ataque potente frontal e menos após o ataque potente na diagonal fechada e o ataque explorando o bloqueio - block out. $\mathrm{O}$ erro de ataque ocorreu mais do que o esperado após o ataque potente na paralela e menos do que o esperado após o ataque explorando o bloqueio - block out.

\section{DISCUSSÃO}

O presente estudo teve como objetivo analisar a relação do tempo e do tipo de ataque com o efeito do ataque na categoria juvenil masculina de alto rendimento competitivo.

$\mathrm{O}$ ataque potente mostra ser o tipo de ataque que mais propicia o ponto? . Esta tendência corrobora Nishijima et al.12, que analisaram um campeonato masculino intercolegial e verificaram que o ataque apresenta poder preditor no resultado do jogo, verificando que o "ataque após a defesa" constituía o fator diferencial entre o sucesso e o insucesso no jogo.

Nos resultados encontrados, relativamente ao tempo de ataque, verificou-se que o $1^{\circ}$ e $2^{\circ}$ tempo de ataque associaram-se significativamente ao ponto, sendo que o $1^{\circ}$ tempo permitiu que ocorresse menos bloqueio da equipe adversária, apesar do $2^{\circ}$ tempo de ataque ter sido mais utilizado. Este fato confirma a tendência do jogo ser cada vez mais rápido no Voleibol atual ${ }^{19}$, estendendo-se esta constatação com os resultados do presente estudo, a equipes de base de alto nível de rendimento competitivo. Este fato deve-se, por certo, a maior utilização de bolas de $1^{\circ}$ tempo em áreas mais centrais da rede (zona 3) e contra bloqueio simples, enquanto as demais zonas de ataque (ataques de zona 4, zona 2 e fundo) estão sujeitas ao tempo de ataque intermediário e contra bloqueios duplos ${ }^{18}$.

Estes resultados confirmam os do estudo de Rocha e Barbanti ${ }^{17}$, que ao analisarem o Voleibol Masculino adulto de alto nível, perceberam que a maioria dos ataques realizados foi de $2^{\circ}$ tempo, sendo que os ataques de $1^{\circ}$ e $2^{\circ}$ tempo culminaram, na maior parte das vezes, em ponto. Do mesmo modo, Papadimitriou et $\mathrm{al}^{16}{ }^{16}$, ao analisarem o Campeonato Grego Masculino, verificaram que a maioria dos ataques foi de $2^{\circ}$ tempo, sendo que o ataque culminou em ponto na maioria das vezes. Deste modo, na medida em que o jogo rápido foi requerido, induziu dificuldades na estruturação do jogo defensivo, nomeadamente ao nível da formação do bloqueio e no posicionamento da defesa baixa.

A investigação tem mostrado, que a velocidade do ataque está também dependente da qualidade de recepção, ou seja, do $1^{\circ}$ toque da equipe após a ação do serviço. Zetou et al. ${ }^{20}$, ao analisarem trinta e oito Jogos Olímpicos Masculinos, perceberam que a boa qualidade da recepção propiciou maior organização ofensiva, permitindo um ataque mais agressivo através de bolas rápidas ( $1^{\circ}$ tempo), o que concomitantemente dificultou a defesa do adversário.

$\bigcirc$ presente estudo mostra que, jogar com menos velocidade resultou em maior continuidade do jogo, devido a melhor estruturação defensiva da 
equipe adversária, restringindo as possibilidades de sucesso da equipe atacante. Sendo assim, o tempo de ataque mais rápido possibilitou maior ocorrência do ponto, enquanto que o tempo de ataque mais lento favoreceu a continuidade do jogo e o erro do ataque. Além disso, constatou-se que, no Voleibol Masculino, jogar com velocidade torna-se necessário desde as categorias de base, devendo este ser um indicativo para o treino de forma a aumentar a agressividade ofensiva das equipes para maior importância conferida à velocidade do jogo.

No que se refere ao tipo de ataque, notou-se que os ataques potentes culminaram mais em ponto de ataque, bem como os ataques explorando o bloqueio (block out e costura), apesar do seu parco recurso. Contrariamente, os ataques colocados favoreceram a organização do sistema defensivo e, consequentemente, o contra-ataque organizado. Ressalta do presente estudo que as equipes de base de alto nível pela necessidade eminente de sobreporem o ataque ao sistema defensivo, pelo recurso ao ataque potente o qual imprimiu maior velocidade e agressividade, induziram a diminuição do tempo de reação do jogador defensor, favorecendo a conquista do ponto de ataque.

Estas tendências corroboram o estudo de Castro e Mesquita ${ }^{18}$, no qual se analisaram doze jogos da Liga Mundial Masculina e seis jogos referentes à fase final do Campeonato da Europa Masculino de 2005 em equipes adultas. Os autores verificaram que os tipos de ataque em que não se verificou toque no bloqueio (forte e colocado) ocorreram mais vezes com $67 \%$ e $12,9 \%$, respectivamente. Enquanto isso, o ataque block out ocorreu 10,5\% das vezes e o ataque costura apresentou 9,6\% das ocorrências. Sendo assim, ficou claro o predomínio do ataque forte, evidenciando uma tendência clara do voleibol de alto rendimento masculino independentemente da categoria de referência (de base ou adulto). Ao assumir-se como regularidade esta tendência, é crucial no treinamento a inclusão desde a formação do ataque rápido, obviamente, desde que os jogadores estejam capazes de o realizar corretamente(estejam capacitados para realizá-lo...), em sintonia com o recurso a estratégias variadas de finalização, quer no que concerne à direção do ataque, quer na exploração do bloqueio.

\section{CONCLUSÕES}

$\mathrm{O}$ ataque apresentou-se como o procedimento de jogo que resultou, na maioria das vezes, em ponto. Ao ser analisado o tempo de ataque, observou-se que houve predominância de um jogo com velocidade intermediária ( $2^{\circ}$ tempo), sendo que, quando o jogo foi efetuado com mais velocidade, as possibilidades de obtenção de sucesso no ataque aumentaram; quando se jogou com menos velocidade ( $3^{\circ}$ tempo), maiores foram as chances de continuidade. $\mathrm{O}$ tipo de ataque mais realizado foi o ataque potente, dada a necessidade de se sobrepor a um sistema defensivo agressivo (bloqueio e defesa) para a conquista do ponto.

É importante, no futuro, estudar outros momentos de jogo, como seja, as condições de partida (recepção e de distribuição), e ainda, a natureza da oposição, pelo recurso a modelos dinâmicos do estudo de performance, no sentido de se obter informação relevante para o treinamento e para a futura pesquisa.

\section{REFERÊNCIAS BIBLIOGRÁFICAS}

10. Borrie A, Jonsson G, Magnusson M. Temporal pattern analysis an dits applicability in sport : an explanation and exemplar data. J Sports Sci 2002;20(10):845-52.

11. Duthie G, Pyne D, Hooper S. Applied physiologie and game analysis of rugby union. Sports Med 2003;33(13):973-91.

12. Lees, A. Technique analysis in sports: a critical review. J Sports Sci 2002;20(10):813-28.

13. Hughes MD. Computerized notation analysis in field games. Ergonomics 1988;31(11):1585-92

14. Gréhaigne J, Bouthier D, Gobdout P. Performance assessment in team sports. J Teach Phys Educ 1997;15(4):500-16.

15. Hughes M, Bartlett R. The use of performance indicators in performance analysis. J Sports Sci 2002; 20(10):739-54.

16. Eom HJ, Schutz RW. Statistical analysis of volleyball team performance. Res Q Exerc Sport 1992;63(1):11-8.

17. Eom HJ, Schutz RW. Transition play in team performance of volleyball: A log linear analysis. Res Q Exerc Sport 1992;63(3):261-9.

18. Grgantov Z, Dizdar D, Jankovic V. Structural analysis of the volleyball game elements based on certain anthropological features. Kinesiology 1998;30(1):44-51.

19. Häyrinen M, Hoivala T, Blomqvist M. Differences between winning and losing teams in men's European top-level volleyball. In: O’Donoghue P, Hughes M, editors Performance Analysis of Sport VI, Cardiff: Center for Performance Analysis, School of Sport, Physical Education and Recreation, University of Wales Institute Cardiff; 2004. p. 194-199.

20. Palao JM, Santos JA, Ureña A. Effect of team level on skill performance in volleyball. Int J Perform Anal Sport 2004;4(2):50-60.

21. Nishijima T, Ohsawa S, Matsuura Y. The relationship between the game performance and group skill in volleyball. Int J Phys Educ 1987;24(4):20-6.

22. Yiannis L, Panagiotis K. Evolution in men's volleyball skills and tactics as evidenced in the Athens 2004 
Olympic Games. Int J Perform Anal Sport 2005;5(2):1 8 .

23. Mesquita I. Modelação do treino das habilidades técnicas nos jogos desportivos. In: Garganta J, editor. Horizontes e órbitas no treino dos jogos desportivos. Porto; 2000. p.73-89.

24. Katsikadelli A. A comparative study of the attack serve in high-level Volleyball Tournaments. J Hum Mov Studies 1996;30(6):259-67.

25. Papadimitriou K, Pashali E, Sermaki I, Mellas S, Papas, M. The Effect of the opponent's serve on the Offensive Actions of Greek of Setters in Volleyball games. Int J Perform 2004;4(1):23-33.

26. Rocha CM, Barbanti VJ. Uma análise dos fatores que influenciam o ataque no Voleibol masculino de alto nível. Rev Bras Educ Fís Esportes 2004;18(4):303-14.

27. Castro J, Mesquita I. Estudo das implicações do espaço ofensivo nas características do ataque no Voleibol masculino de elite. Rev Port Ci Desporto 2008;8(1):114-25.

28. Fröhner B. Select aspects of developments in women's volleyball. Coach 1997;1:6-19.

29. Zetou E, Moustakidis A, Tsigilis N, Komninakidou A. Does effectiveness of skill in complex I predict win in men's olympic volleyball games. J Quant Anal Sports 2007;3(4):1-9.

30. César B, Mesquita I. Caracterização do ataque do jogador oposto em função do complexo do jogo, do tempo e do efeito do ataque: estudo aplicado no Voleibol feminino de elite. Rev BrasEduc Fís Esporte 2006;20(1):59-69.
31. Dias C. A distribuição no Voleibol: aplicar uma estratégia eficaz. Treino Desportivo 2004;6(24):51-59.

32. Weishoff P. Attacking. In: Don S, Cecile R. (Eds.). The Volleyball Coaching Bible. USA: Human Kinetics; 2002. p 199-226.

33. Selinger A, Ackermann-Blount J. Arie Salinger's power volleyball. New York: St. Martin Press, 1986.

34. Tabachnick B, Fidell L. Using Multivariate Statistics. New York: Harper \& Row Publishers, 1989.

35. Fleiss JL. Statistical Methods for Rates and Proportions (2 Ed.). Wiley-Interscience, 1981.

\section{Endereço para correspondência}

Gustavo De Conti Teixeira Costa, Rua Dr. Juvenal dos Santos, 431/apto 101, Bairro Luxemburgo, CEP: 30380-530 - Belo Horizonte/ MG / Brasil. E-mail: conti02@hotmail.com 